\title{
Inovação no setor público: análise da produção científica em periódicos nacionais e internacionais da área de administração
}

\author{
Soraya Monteiro Brandão \\ Escola Nacional de Administração Pública \\ Maria de Fátima Bruno-Faria \\ Universidade de Brasilia
}

\begin{abstract}
O artigo tem como objetivo apresentar um panorama da produção científica em periódicos nacionais e internacionais sobre inovação no setor público no período entre 2000 e 2010 . Foram analisados os objetivos dos estudos, as opções metodológicas assumidas pelos autores e as principais contribuições para o avanço da compreensão do tema. No Brasil, a produção em tais periódicos encontra-se em estágio inicial, mas constata-se um número crescente de publicações nos últimos cinco anos. Já no contexto internacional, a produção científica encontra-se mais consolidada, em três principais focos de estudo: fatores ambientais, organizacionais e gerenciais que influenciam a inovação no setor público. Foram identificadas lacunas de pesquisa em cinco temas: (a) processo de inovação, (b) indutores e barreiras, (c) características das lideranças que facilitam a inovação, (d) efeitos da inovação e (e) fatores que contribuem para a disseminação de inovações no setor público.
\end{abstract}

Palavras-chave: inovação; setor público; administração pública.

Investigaciones sobre innovación en el sector público: análisis de la literatura científica en revistas nacionales e internacionales en el ámbito de la administración

El artículo tiene como objetivo presentar una visión general de la literatura científica en revistas nacionales e internacionales sobre innovación en el sector público entre 2000 y 2010. Se analizaron los objetivos de las investigaciones, las opciones metodológicas asumidas de los autores y las principales contribuciones al avance de la comprensión del tema. En Brasil, la producción en dichas revistas se encuentra en sus primeras etapas, pero ha habido un número creciente de publicaciones en los últimos cinco años. Ya en el contexto internacional, la producción científica está más consolidada en tres focos principales de estudio: factores ambientales, organizacionales y gerenciales que influyen en la innovación en el sector público. Se identificaron cinco temas para investigación futura: (a) innovación de procesos, (b) inductores y obstáculos, (c) características de los líderes que faciliten la innovación, (d) efectos de la innovación y (e) factores que contribuyen a la propagación de innovaciones en el sector público.

Palabras clave: innovación; sector público; administración pública.

Artigo recebido em 19 abr. 2012 e aceito em 5 out. 2012.

Rev. Adm. Pública - Rio de Janeiro 47(1):227-248, jan./fev. 2013 


\begin{abstract}
Research on public sector innovation: analysis of scientific literature in national and international journals in the area of administration

This article aims to present an overview of scientific literature in national and international journals on innovation in the public sector between 2000 and 2010. The objectives of the studies, the methodological choices assumed by the authors and major contributions to the advancement of understanding were analyzed. In Brazil, the production in such journals is in its early stages, but there has been a growing number of publications in the past five years. In the international context, the scientific production is more consolidated in three main focuses of study: environmental, organizational and managerial factors influencing innovation in the public sector. Research gaps were identified in five themes: (a) innovation process, (b) drivers and barriers, (c) characteristics of leaders that facilitate innovation, (d) effects of innovation and (e) factors that contribute to the dissemination of innovations in the public sector.
\end{abstract}

KEY WORDs: innovation; public sector; public administration.

\title{
1. Introdução
}

Governos em todo o mundo têm estado sob permanente pressão da sociedade para responder às demandas dos cidadãos e à crescente complexidade de seus ambientes (Alberti e Bertucci, 2006). De acordo com Schwella (2005), a globalização, o combate à desigualdade, o respeito à diversidade e a busca pela boa governança e por uma gestão pública eficiente são alguns dos desafios enfrentados pelos governos no século XXI que exigem criatividade e abordagens inovadoras.

Do ponto de vista econômico, Potts e Kastelle (2010) identificam três razões para estimular a inovação no setor público: a primeira é que, na maioria dos países da Organização para Cooperação Econômica e Desenvolvimento (OCDE), o setor público é um componente significativo da macroeconomia, contribuindo com parcela significativa do PIB (entre 20 e $50 \%$ ). A segunda é que o setor público é responsável por prover serviços para cidadãos e empresas e a inovação pode apoiar o alcance de melhores resultados por meio de novas maneiras de resolver problemas. A terceira é que, considerando os sistemas nacionais de inovação, uma grande razão para promover a inovação no setor público é sua responsabilidade sobre a definição das políticas de fomento à inovação no setor privado.

Do ponto de vista social, um setor público inovador que oferece produtos e serviços de boa qualidade (facilidade de uso, acesso, oportunidade) pode garantir uma relação mais eficaz com os cidadãos. Vigoda-Gadot e colaboradores (2008) afirmam que a eficiência do setor público de um país e uma prestação de serviços públicos de boa qualidade são vitais para alcançar operações mais transparentes, podendo resultar em maior satisfação dos usuários com os serviços oferecidos e em maior nível de confiança no setor público.

É nesse contexto que a inovação vem ganhando importância como tema estratégico para a administração pública (Mulgan e Albury, 2003; Walker, 2007; Vigoda-Gadot et al., 2008), fazendo com que cada vez mais estudos se dediquem à análise deste fenômeno de forma empírica. Embora crescente, Walker (2007) argumenta, porém, que a produção científica 
ainda se mantém limitada nesse campo e que, portanto, se faz necessário que pesquisadores desenvolvam mais conhecimento sobre a natureza da inovação no serviço público.

Assim, objetiva-se neste artigo analisar a produção científica sobre inovação no setor público na área de administração, constante dos periódicos nacionais e internacionais da área de administração, a fim de identificar lacunas de pesquisa sobre o tema que possam contribuir para o avanço do conhecimento e para estimular sua prática no contexto brasileiro. Para isso, este artigo foi organizado em cinco partes. A primeira apresenta o marco teórico sobre inovação que será utilizado nas análises das seções seguintes. A segunda descreve o método utilizado nesta revisão bibliográfica. A terceira organiza uma síntese dos temas e dos principais resultados, enquanto a quarta parte analisa e discute os estudos revisados a partir de três categorias de análise. Por fim, a última parte apresenta as principais conclusões e as lacunas de pesquisa identificadas.

\section{Referencial teórico}

Os primeiros estudos sobre inovação buscavam explicar a relação entre inovação tecnológica e desenvolvimento econômico e focavam o desenvolvimento de produtos e processos com aplicação comercial no setor privado. Schumpeter (1982) foi um dos pioneiros a relacionar desenvolvimento econômico com inovações tecnológicas. Em sua teoria, a inovação é descrita como novas combinações entre materiais e forças produtivas a fim de viabilizar novos produtos e o desenvolvimento econômico, e esse processo dinâmico de substituição de antigas tecnologias por novas é denominado "destruição criadora".

A partir da ampliação do interesse no estudo da inovação em outros setores, novas definições foram ampliando seu escopo, descrevendo também inovações sociais, inovações em serviços e inovações no setor público. Neste contexto, Van de Ven, Angle e Poole (2000) definem inovação como o processo de desenvolver e implementar novas ideias. Os autores destacam ainda que, de uma perspectiva gerencial, o processo de inovação consiste em motivar e coordenar pessoas para desenvolver e implementar novas ideias por meio do engajamento (ou relacionamentos) com outros e fazendo adaptações necessárias para atingir os resultados desejados no contexto de mudanças institucionais e organizacionais.

Rogers (1995:11) define inovação como uma "ideia, prática ou objeto percebido como novo pelo indivíduo ou pela unidade adotante". É uma definição bastante ampla, destacando principalmente o grau de novidade da inovação do ponto de vista de quem a adota.

Para a Organização para Cooperação Econômica e Desenvolvimento (OCDE) (2005:55), “inovação é a implementação de um produto (bem ou serviço) novo ou significativamente melhorado, ou um processo, ou um novo método de marketing, ou um novo método organizacional nas práticas de negócios, na organização do local de trabalho ou nas relações externas". Aqui o conceito de inovação se baseia em quatro tipos de inovação: produto, processo, marketing ou organizacional. 
São quatro os tipos de inovação identificados pela OCDE (2005:57):

1) Inovação de produto: é a introdução de um bem ou serviço novo ou significativamente melhorado no que concerne a suas características ou usos previstos.

2) Inovação de processo: é a implementação de um método de produção ou distribuição novo ou significativamente melhorado. Incluem-se mudanças significativas em técnicas, equipamentos e/ou softwares.

3) Inovação de marketing: é a implementação de um novo método de marketing com mudanças significativas na concepção do produto ou em sua embalagem, no seu posicionamento, em sua promoção ou na fixação de preços.

4) Inovação organizacional: é a implementação de um novo método organizacional nas práticas de negócios da empresa, na organização do seu local de trabalho ou em suas relações externas.

Outros autores também se dedicaram a distinguir conceitualmente os diferentes tipos de inovação. Para Sundbo (2003), a inovação de produto refere-se à introdução de novos produtos ou serviços no mercado. Já Gallouj (2007) propõe uma abordagem integradora que possa reportar a inovação tecnológica e não tecnológica, tanto nos serviços como nos bens.

Outro tipo de inovação comumente estudado no âmbito do setor privado é a inovação de processo, a qual pode ser entendida como a introdução de novos processos de produção de produtos ou serviços, tais como os gerados por novas tecnologias ou novas rotinas de trabalho (Sundbo, 2003; Carayannis, Gonzalez e Wetter, 2003).

A literatura também se refere à inovação em gestão, administrativa ou organizacional. Embora possa haver sutis diferenças semânticas entre tais termos, este artigo optou por tratálos como sinônimos. Birkinshaw, Hamel e Mol (2008:829) revisaram a literatura em inovação em gestão e a definiram operacionalmente como "a geração e implementação de práticas gerenciais, processos, estrutura ou técnicas que são novas para o estado da arte e que pretendem potencializar os objetivos organizacionais". Segundo os autores, a inovação em gestão é concebida como um construto multidimensional que inclui processos estruturantes, operacionais e administrativos. Além disso, como o grau de novidade é definido como o estado da arte, essas inovações são radicais e sua adoção pode resultar em mudanças mais significativas nos sistemas e processos gerenciais da organização.

Já para Carayannis, Gonzalez e Wetter (2003) a inovação administrativa é compreendida como mudanças nas características dos elementos organizacionais e institucionais, como políticas, estrutura ou alocação de recursos, entre outros.

Na próxima seção são apresentados os critérios de seleção dos estudos revisados e as categorias de análise utilizadas nesta revisão bibliográfica. 


\section{Método}

Para isso, adotaram-se no levantamento bibliográfico como critérios de seleção dos artigos científicos (a) aqueles que, de acordo com a classificação Thomson Scientific ISI, possuíam em 2011 escore acima de 1, em razão de o Fator de Impacto publicado anualmente pela Thomson ISI (antigo Institute for Scientific Information) no Journal Citation Reports vir sendo utilizado mundialmente como parâmetro de avaliação da relevância da produção científica; (b) foram também levantados artigos em periódicos nacionais da área de administração classificados pela Capes, na base de dados Qualis 2008, nos estratos mais elevados (A e B), que refletem também o impacto dessa produção; (c) além disso, foram pesquisadas as bases de dados Sage, Willey, Oxford Journals, Atypon, JSTOR, Proquest e Scielo porque abarcam grande número de periódicos internacionais, a fim de se realizar um levantamento o mais abrangente possível de artigos científicos sobre o tema; (d) como critério temporal de seleção do material por meio de levantamento bibliográfico, optou-se pelo período compreendido entre 2000 e 2010 por incluir as produções mais recentes sobre o tema; (e) foram adotados os seguintes quatro descritores: inovação e setor público; inovação e público; inovação em gestão; inovação em gestão pública, e os termos correspondentes em inglês.

Após a leitura dos resumos, foram descartados aqueles que não tratavam de inovação no setor público ou que não atendiam aos critérios de classificação definidos anteriormente. Foram aproveitados 30 artigos, sendo sete publicados em periódicos nacionais e 23 em periódicos internacionais. Devido ao pequeno número de relatos de pesquisa encontrados sob os critérios anteriormente definidos, foram incluídos também relatórios de pesquisas não empíricas sobre o tema, totalizando 34 estudos revisados.

Os estudos sobre inovação no setor público foram confrontados com o referencial teórico sobre inovação no setor privado e analisados de acordo com três categorias, a fim de identificar as abordagens adotadas pelos autores. A primeira categoria refere-se ao conceito de inovação no setor público utilizado nas pesquisas. A segunda categoria buscou comparar os tipos de inovação estudados no setor público em relação aos mais comumente estudados no setor privado. Por fim, a terceira categoria buscou classificar os artigos quanto ao método de pesquisa adotado (qualitativo ou quantitativo).

\section{Síntese dos estudos revisados}

Esta seção apresenta um resumo das principais pesquisas sobre inovação no setor público realizadas entre 2000 e 2010 a fim de evidenciar as contribuições teóricas e empíricas para o tema, bem como evidenciar as lacunas de pesquisa existentes. O quadro 1 identifica os 30 artigos revisados que foram publicados em periódicos nacionais e internacionais de administração. 


\section{Trabalhos publicados sobre inovação no setor público em periódicos nacionais e internacionais}

\begin{tabular}{|c|c|c|c|c|}
\hline Classificação & Periódicos & Pontuação & Artigos & Autores \\
\hline Capes A & Revista de Administração Pública & $\mathrm{A} 2$ & 1 & Queiroz e Ckagnazaroff (2010) \\
\hline \multirow[t]{5}{*}{ Capes B } & Revista Organizações \& Sociedade & B2 & 1 & Farah (2008) \\
\hline & $\begin{array}{l}\text { International Public Management } \\
\text { Review }\end{array}$ & B2 & 1 & Bommert (2010) \\
\hline & Revista do Serviço Público & B4 & 2 & Schwella (2005); Asazu (2006) \\
\hline & Cadernos Enap & B4 & 3 & $\begin{array}{l}\text { Ferrarezi, Amorim e Tomacheski } \\
\text { (2010); Vargas (2010); Ferrarezi e } \\
\text { Amorim (2007) }\end{array}$ \\
\hline & $\begin{array}{l}\text { Innovation: Management, Policy \& } \\
\text { Practice Journal }\end{array}$ & B4 & 6 & $\begin{array}{l}\text { Luke, Verreynne e Kearins (2010); } \\
\text { Potts (2010); Potts e Kastelle } \\
\text { (2010); Gambarotto e Camozzo } \\
\text { (2010); Potts (2009); Husig e Mann } \\
\text { (2010) }\end{array}$ \\
\hline \multirow[t]{8}{*}{ ISI (1 a 3) } & Journal of Management Studies & 2,805 & 1 & $\begin{array}{l}\text { Damanpour, Walker e Avellaneda } \\
\text { (2009) }\end{array}$ \\
\hline & $\begin{array}{l}\text { Journal of Public Administration } \\
\text { Research and Theory }\end{array}$ & 1,776 & 4 & $\begin{array}{l}\text { Damanpour e Schneider (2009); } \\
\text { Walker, Damanpour e Devece } \\
\text { (2010); Walker (2007); Lonti e } \\
\text { Verma (2003) }\end{array}$ \\
\hline & $\begin{array}{l}\text { International Journal of Technol- } \\
\text { ogy Assessment in Health Care }\end{array}$ & 1,794 & 1 & Dobbins et al. (2001) \\
\hline & British Journal of Social Work & 1,524 & 1 & Brown (2010) \\
\hline & British Journal of Management & 1,448 & 1 & Damanpour e Schneider (2006) \\
\hline & Public Administration Journal & 1,292 & 5 & $\begin{array}{l}\text { Hansen (2010); Fernández e Wise } \\
\text { (2010); Vigoda-Gadot et al. (2008); } \\
\text { Walker (2006); Walker, Jeanes e } \\
\text { Rowland (2002) }\end{array}$ \\
\hline & Public Administration Review & 1,141 & 2 & $\begin{array}{l}\text { Borins (2000); Tolbert, Mossberger } \\
\text { e Mcneal (2008) }\end{array}$ \\
\hline & $\begin{array}{l}\text { The American Review of Public } \\
\text { Administration }\end{array}$ & 1,000 & 1 & Borins (2001b) \\
\hline Total & 14 periódicos & & 30 & 46 autores \\
\hline
\end{tabular}

Fonte: Elaborado pelas autoras. 
Verificou-se que os periódicos com mais trabalhos publicados sobre inovação no setor público foram o Innovation: Management, Policy \& Practice Journal, com seis artigos, e o Public Administration Journal, com cinco artigos. Em seguida, tem-se o Journal of Public Administration Research and Theory, com quatro artigos, Cadernos Enap, com três artigos, Revista do Serviço Público e Public Admnistration Review, com dois artigos cada um, e os demais oito periódicos com apenas um artigo cada um.

Além dos estudos científicos apresentados anteriormente, foram revisados também estudos realizados por agências governamentais e institutos de pesquisa que buscaram caracterizar a inovação no setor público em seus países, conforme quadro 2.

$$
\text { Quadro } 2
$$

Estudos realizados por agências governamentais e institutos de pesquisa

\begin{tabular}{|lll|}
\hline Países & \multicolumn{1}{c|}{ Pesquisa } & \multicolumn{1}{c|}{ Autores } \\
\hline $\begin{array}{l}\text { 27 países-membros da União Europeia, } \\
\text { Noruega e Suíça }\end{array}$ & $\begin{array}{l}\text { Innobarometer on innovation in public } \\
\text { administration }\end{array}$ & The Gallup Organization (2010) \\
Reino Unido & $\begin{array}{l}\text { Achieving innovation in central } \\
\text { government organizations }\end{array}$ & National Audit Office (2006) \\
Dinamarca, Finlândia, Islândia, Noruega & $\begin{array}{l}\text { Measuring public innovation in the } \\
\text { nordic countries }\end{array}$ & Bloch (2010) \\
e Suécia & $\begin{array}{l}\text { Innovation in public sector } \\
\text { organisations }\end{array}$ & Hughes, Moore e Kataria (2011) \\
\hline
\end{tabular}

Fonte: Elaborado pelas autoras.

Os resultados dos principais estudos revisados neste levantamento bibliográfico serão sintetizados a seguir, agrupados em três categorias: teóricos, empíricos e não científicos.

Nos ensaios teóricos, os principais temas abordados nos estudos revisados foram a relação entre liderança e inovação, disseminação de inovações e a discussão sobre a utilização de experimentos como forma de alavancar a inovação no setor público. Schwella (2005), por exemplo, defende que enfrentar o conjunto de questões geradas por fenômenos complexos e dinâmicos associados à inovação exige uma abordagem de aprendizagem social da liderança. As pessoas e as organizações precisam ser mobilizadas para um processo de aprendizagem pelo qual irão adaptar seus comportamentos a fim de progredir sob condições de incerteza e turbulência, e isso exige diferentes competências de liderança como reflexão e análise, associadas a competências de facilitação.

Farah (2008), por sua vez, defende que a disseminação de inovações no contexto do poder local no Brasil depende de características intrínsecas da política ou do programa e da relação entre a inovação e o contexto em que ela será implementada (influência de atores e instituições externas e internas). Os fatores apontados pela autora como determinantes da disseminação de inovações em governos locais são: (i) a capacidade que uma política ou processo tem de consti- 
tuir-se em solução inovadora para problemas sociais; (ii) a natureza do problema enfrentado (se é comum a outras localidades); (iii) se outros municípios percebem o problema como relevantes para seu contexto específico; (iv) a convergência entre o problema tratado pela inovação e a agenda de políticas públicas de diferentes localidades; (v) o acesso à informação (a disseminação depende da difusão de informações); e (vi) o papel ativo desempenhado por atores sociais e políticos na seleção de inovações nas localidades adotantes.

Já Potts $(2009$, 2010) elaborou alguns artigos sobre a importância da utilização de experimentos como método para estimular a inovação. Potts (2009) argumenta que o déficit de inovação do setor público em relação ao setor privado pode ser explicado como uma consequência não intencional da busca pela eliminação do desperdício por meio da eficiência, accountability e transparência. A questão destacada pelo autor é de que esta pode ser uma falsa eficiência, dado que também elimina o "bom desperdício", que por sua vez é um custo necessário da experimentação.

Ainda sob o argumento de promover experimentos para fomentar a inovação, Potts (2010) destaca que a inovação no setor público é comumente vista como em prol de fazer coisas novas e melhores, mas mantendo o que já está feito. Em outras palavras, uma agenda de inovação no setor público é vista primordialmente como criação e não como destruição. Sob o argumento de que novos programas de políticas públicas são fáceis de começar (inovar) e difíceis de serem extintos (eliminados), Potts propõe uma abordagem à inovação no setor público que se concentre na destruição e não na criação por meio de uma metodologia sistemática e cientificamente rigorosa de seleção e de avaliação das implicações da retirada de determinados programas, políticas, regras ou mecanismos já existentes.

Por fim, Potts e Kastelle (2010) sugerem uma nova agenda de pesquisa para a inovação no setor público que se concentre menos sobre a imitação de boas práticas do setor privado ou de outras organizações públicas e mais em métodos científicos de experimentos controlados randomizados. Os autores argumentam que o setor público deveria agir sobre conhecimentos previamente testados e não sobre hipóteses. Que deveria experimentar novas ideias em ambientes controlados para minimizar danos não intencionais em vez de buscar maximizar o valor agregado esperado. Assim, os autores concluem que o modelo de inovação para o setor público deveria se basear em experimentos científicos bem construídos e suas consequências, em vez de em aventuras empreendedoras bem gerenciadas.

No que diz respeito às pesquisas empíricas revisadas, um tema com um grande número de publicações em periódicos científicos é a identificação de antecedentes organizacionais, ambientais ou gerenciais que facilitam ou inibem a adoção de inovações.

Damanpour e Schneider (2006), por exemplo, examinaram efeitos de características ambientais, organizacionais e gerenciais em diferentes fases do processo de inovação (iniciação, decisão de adoção e implementação) em 1.200 organizações públicas nos Estados Unidos. As autoras concluem que características organizacionais e atitudes pró-inovação de dirigentes influenciam mais fortemente a adoção de inovações que características ambientais ou características demográficas de dirigentes. 
Outro estudo foi desenvolvido por Walker (2007) em organizações públicas locais do Reino Unido e buscou verificar que antecedentes ambientais e organizacionais e que tipos de inovação adotados simultaneamente resultam em níveis de inovação organizacional mais altos. Os resultados da pesquisa de Walker demonstraram que diferentes combinações de antecedentes ambientais e organizacionais importam para a inovação e que a relação complementar entre diferentes tipos de inovação não é tão comum quanto teorizada.

Damanpour e Schneider (2009) buscaram compreender a relação entre características da inovação, perfil dos gerentes e a adoção de inovações em 725 organizações públicas de governos locais nos Estados Unidos. As autoras identificaram que tanto características da inovação quanto dos gerentes influenciam a adoção de inovações. No entanto, não foram evidenciados efeitos moderadores significativos das características dos gerentes na relação entre características da inovação e sua adoção. Também foi verificado por Damampour e Schneider (2009) que o nível educacional dos gerentes afeta positivamente a inovação, corroborando Kearney, Feldman e Scavo (2000), que afirmam que, por meio de melhor educação, gerentes mais especializados e com maior capacidade intelectual beneficiam a inovação em organizações. Já tempo no cargo, de acordo com Damampour e Schneider (2009), apresentou um efeito não linear, indicando que a experiência gerencial afeta positivamente a adoção da inovação até o ponto em que diminui a propensão do gerente em promover mudanças nas práticas e rotinas organizacionais existentes com as quais ele já se acostumou.

Outros temas também vêm ganhando atenção de pesquisadores nacionais e internacionais. Vigoda-Gadot e colaboradores (2008) investigaram a inovação no setor público em oito países da Europa, analisando a relação entre antecedentes e resultados da inovação da perspectiva do cidadão. Os resultados da pesquisa revelaram que (i) capacidade de resposta, liderança e visão são os antecedentes mais influentes da inovação no setor público; (ii) a inovação afeta a confiança e a satisfação com a administração pública; e (iii) que o efeito da inovação no setor público na confiança e na satisfação é mediado ou diretamente relacionado à imagem da organização.

Já Damanpour, Walker e Avellaneda (2009) focaram seu estudo nas consequências da adoção de três tipos de inovação (serviços, processos tecnológicos e processos administrativos) em organizações públicas de serviços. A tese principal estudada foi que o impacto da inovação no desempenho organizacional depende da composição de diferentes tipos de inovação ao longo do tempo. Os resultados dessa pesquisa demonstraram, no entanto, que o foco na adoção de tipos específicos de inovação todos os anos é prejudicial e que a consistência em adotar a mesma composição de tipos de inovação ao longo dos anos não tem efeito sobre o desempenho organizacional.

Walker, Damanpour e Devece (2010), por sua vez, investigaram o papel mediador da gestão do desempenho no desempenho organizacional. Os principais achados da pesquisa são que: (i) inovação em gestão não tem impacto direto no desempenho organizacional; (ii) a gestão do desempenho afeta diretamente o desempenho organizacional; e (iii) o efeito da inovação em gestão no desempenho organizacional é mediado pela gestão do desempenho. Os autores destacam, portanto, que para obter os melhores resultados pela introdução de 
inovações em gestão faz-se necessário assegurar que estruturas apropriadas de gestão do desempenho estejam implementadas, porque, sem elas, as melhorias no desempenho da organização podem não se materializar.

Outro aspecto importante explorado em pesquisas empíricas é o papel que os líderes exercem na inovação. Fernández e Wise (2010) e Hansen (2010) concluíram em suas pesquisas que gerentes que enxergam seu papel como agentes de mudança em seu trabalho diário afetam positivamente a adoção de inovações. Borins (2001a) corrobora esse achado ao afirmar que, quando inovações são originadas por gerentes do nível intermediário ou de linha de frente, o suporte de dirigentes e líderes é essencial para o sucesso da inovação.

Outro tema que tem recebido atenção de pesquisadores é a caracterização de inovações premiadas em concursos. Borins (2001a, 2001b, 2002) realizou pesquisas sobre inovação nos Estados Unidos, Canadá e outros países do Commonwealth a fim de analisar as principais características das inovações inscritas em prêmios. O autor conclui que, embora o setor público tradicionalmente seja considerado não receptivo a inovações, um crescente conjunto de evidências revela que gerentes de nível intermediário e pessoal de linha de frente são responsáveis por muitas inovações.

No Brasil, algumas pesquisas têm buscado estudar a inovação a partir da análise de iniciativas premiadas no Concurso Inovação na Gestão Pública Federal, promovido pela Escola Nacional de Administração Pública (Enap) e pelo Ministério do Planejamento, Orçamento e Gestão (MPOG). Ferrarezi e Amorim (2007:33), por exemplo, concluíram que "o Concurso ofereceu contribuições para a transformação da administração pública federal brasileira, tanto pela sua abrangência, envolvendo a maior parte das instituições do Executivo federal, quanto pela qualidade das iniciativas inovadoras premiadas". Nos três períodos analisados foi possível perceber uma relação entre o foco gerencial de cada governo e as iniciativas premiadas: o período de 1996 a 1998, mais gerencialista; o período de 1999 a 2002, com gestão empreendedora e revitalização e integração do planejamento e orçamento governamental; e o período de 2003 a 2006, direcionado para o estímulo da coordenação, otimizadora de políticas públicas prioritárias.

Já a pesquisa de Ferrarezi, Amorim e Tomacheski (2010:46-49) identificou algumas condições que favorecem a sustentabilidade das inovações no governo federal brasileiro. Um dos achados dessa pesquisa é que o ambiente institucional favorável está associado ao estilo de liderança, ao grau de envolvimento das equipes, à utilização de instrumentos gerenciais pela instituição para planejamento, acompanhamento e difusão dos resultados e à constante adaptação da iniciativa às novas circunstâncias, metas e desafios da organização e do governo.

Vargas (2010) também se dedicou à análise de ações premiadas no Concurso da Enap, buscando verificar se as inovações conseguiram se disseminar para outros órgãos ou para outros setores da própria organização. O autor encontrou como principais achados que (i) em 40\% dos casos analisados houve disseminação; (ii) as inovações foram concebidas no interior do próprio órgão responsável e pela própria equipe responsável pela iniciativa; (iii) houve predomínio de iniciativas capitaneadas pela gerência de nível intermediário; (iv) a in- 
serção dos gestores em redes profissionais ou em conselhos temáticos atuou como facilitador da disseminação; (v) houve disseminação de iniciativas que conseguiram se tornar referência para a sociedade e não tiveram sua continuidade ameaçada por mudanças de orientação política; (vi) nos casos em que houve maior identificação entre a ação e as prioridades governamentais, a disseminação foi mais frequente; (vii) houve predomínio de disseminação parcial (apenas parte da iniciativa original foi aproveitada); (viii) o prêmio parece favorecer diretamente a consolidação e a continuidade das experiências e, somente de forma indireta, a disseminação.

Nos últimos anos, outras linhas de pesquisa sobre inovação no setor público vêm sendo conduzidas por governos ou agências estatais. O National Audit Office (2006) do Reino Unido desenvolveu uma pesquisa sobre inovação no governo central, buscando identificar os indutores internos e externos, os potenciais impactos positivos e negativos e as barreiras para a inovação. Em 2010, a Comissão Europeia publicou um relatório da pesquisa realizada pelo Instituto Gallup para medir as estratégias de inovação de organizações públicas europeias (The Gallup Organization, 2010).

Já em 2011, foram publicados diversos relatórios de pesquisas conduzidas por agências estatais sobre inovação no setor público em diversos países. O projeto Measuring innovation in the public sector in the Nordic countries: toward a common statistical approach (Mepin), iniciado em 2008 e concluído em 2011, teve como objetivo desenvolver um modelo de medição para coletar dados sobre inovação no setor público comparáveis internacionalmente (Bloch, 2011). No estudo piloto realizado com organizações públicas da Dinamarca, Finlândia, Islândia, Noruega e Suécia foram pesquisados diversos aspectos, a saber: tipos de inovações mais frequentes; atividades e gastos em inovação; objetivos da inovação; canais de informação para atividades de inovação; cooperação; financiamento externo; práticas inovadoras de compras e aquisições; indutores e barreiras; estratégia, gestão da inovação.

Outra agência governamental interessada no tema é a National Endowment for Science, Technology and the Arts (Nesta), do Reino Unido, que também vem empreendendo estudos desde 2008 a fim de construir índices capazes de capturar as particularidades da inovação em organizações públicas. Em 2011, foi publicado o relatório final da pesquisa piloto que testou um modelo de medição da inovação no setor público baseado em capacidades para inovação, atividades de inovação, impacto no desempenho e condições setoriais para a inovação (Hughes, Moore e Kataria, 2011).

Na próxima seção serão apresentados os resultados da análise dos estudos revisados, de acordo com as categorias definidas na seção de método.

\section{Análise e discussão dos resultados}

Primeiramente, será realizada uma análise dos conceitos de inovação no setor público adotados nas pesquisas revisadas à luz do marco teórico da inovação no setor privado anteriormente apresentado. 
Estudos sobre inovação no setor público são relativamente recentes e, assim como no setor privado, há uma variedade de definições do fenômeno entre os estudiosos do tema. Damanpour e Schneider (2006), Walker (2007) e Damanpour, Walker e Avellaneda (2009) entendem que, no nível organizacional, inovação pode ser definida como o desenvolvimento e a implementação de um novo produto, serviço, processo, tecnologia, política, estrutura ou sistema administrativo. Nessa definição, os tipos de inovação são a base para a compreensão do fenômeno.

Walker, Damanpour e Devece (2010), por sua vez, ampliam esse conceito entendendo a inovação como geração (desenvolvimento) ou adoção (uso) de novas ideias, objetos ou práticas. A geração de inovações resulta em produtos, serviços e práticas que são novas para o estado da arte (ou pelo menos para uma população organizacional) e a adoção resulta no uso de produtos, serviços ou práticas novos para a unidade adotante — seja ele um indivíduo, uma equipe ou a organização propriamente dita.

Farah (2008) apresenta um novo olhar sobre o tema ao buscar compreender a inovação a partir da perspectiva da disseminação. Para a autora, inovação é um novo e bem-sucedido arranjo particular de determinados componentes que pode ser potencialmente útil em outros contextos e localidades, como solução para um problema específico. Já Ferrarezi e Amorim (2007:17), para fins de classificação de iniciativas para o Concurso Inovação na Gestão Pública Federal da Enap, consideram que "inovações são mudanças em práticas anteriores, por meio da incorporação de novos elementos da gestão pública ou de nova combinação dos mecanismos existentes, que produzam resultados significativos para o serviço público e para a sociedade".

De maneira similar, Bloch (2010) e Hughes, Moore e Kataria (2011), buscando adaptar a proposta do Manual de Oslo (OCDE, 2005) para o contexto do serviço público, propõem que a inovação seja vista como a implementação de uma mudança significativa na maneira como a organização opera ou nos produtos que oferta. Para os autores, inovações compreendem serviços, produtos, processos ou métodos novos ou significativamente melhorados, devendo ser novos para a organização, embora possam ter sido desenvolvidos por outros.

Analisando-se as definições apresentadas anteriormente, é possível identificar que, tanto no setor público quanto no privado, o requisito mínimo para se definir uma inovação é que ela seja inédita ou significativamente melhorada para a organização. Isto inclui produtos, processos e métodos que as organizações desenvolvem de maneira pioneira e/ou aqueles que foram adotados de outras empresas ou organizações. Outro aspecto importante que merece destaque é que a ideia, prática ou processo deve ter sido implementado para que possa ser considerado uma inovação.

Outro aspecto a ser analisado diz respeito à autoria ou grau de novidade da inovação. Fernández e Wise (2010) identificam aspectos que diferenciam inovações "inventadas" de inovações "adotadas". Para os autores, o primeiro tipo de inovação envolve a invenção ou descoberta de processos, tecnologias ou produtos inteiramente novos. A ênfase está na originalidade da inovação, indicando que nenhuma outra organização gerou a mesma ideia anteriormente. $\mathrm{O}$ segundo tipo consiste em processos, tecnologias e produtos adotados ou 
implementados pela primeira vez em uma organização, ou seja, são novos apenas para a organização focal, tendo sido inventados em outro lugar. Esses autores, com base em estudos sobre inovações premiadas no Programa de Inovações do Governo Americano, chegaram à conclusão de que a adoção de inovações parece ser mais frequente no setor público do que inovações inventadas ou geradas. Essa constatação parece fazer sentido também no contexto internacional, devido ao grande número de estudos publicados nos últimos anos com foco na adoção de inovações no setor público (Damanpour e Schneider, 2006; Damanpour, Walker e Avellaneda, 2009; Walker, 2007; Walker, Damanpour e Devece, 2010).

Em síntese, esta revisão bibliográfica revela que, apesar de as duas perspectivas de autoria e grau de novidade estarem geralmente contempladas nas definições de inovação (inovações pioneiras ou adotadas) tanto no setor privado quanto no público, o primeiro tende a valorizar mais inovações que se caracterizam pelo ineditismo, enquanto o segundo mais frequentemente adota inovações geradas originalmente em outras organizações. É importante frisar, porém, que isso não implica necessariamente classificar um setor como mais ou menos inovador do que o outro.

A segunda categoria de análise utilizada nesta revisão bibliográfica concentra-se na distinção entre os diferentes tipos de inovação estudados no setor privado e no setor público, levando-se em consideração que eles possuem características diversas e podem ser influenciados por diferentes fatores ambientais e organizacionais.

Walker (2006), por exemplo, define inovações de produto como novos bens ou serviços, sendo, portanto, convergente com a definição proposta por Sundbo (2003), já discutida. A partir de 2007, Walker passa a investigar apenas inovações em serviços, definidas como novos serviços oferecidos por organizações públicas para atender novos usuários externos ou demandas do mercado. $\mathrm{O}$ autor defende, ainda, que a natureza das inovações em serviços pode ser mais bem entendida por meio da relação com os usuários. Assim, Walker identifica e testa três tipos de inovação em serviços: totais, expansionistas e evolucionárias. Inovações totais envolvem oferecer serviços novos para novos usuários. Inovações expansionistas envolvem a oferta de serviços existentes para um novo grupo de usuários. O terceiro tipo, inovações evolucionárias, inclui a oferta de novos serviços para usuários existentes.

Outro tipo de inovação estudado no setor público é a inovação de processo, a qual tem foco interno e busca aumentar a eficiência e a efetividade de processos organizacionais para facilitar a produção e a entrega de produtos ou serviços para os consumidores (Damanpour, Walker e Avellaneda, 2009). Consequentemente, inovações de processo não geram produtos ou serviços para usuários, mas podem influenciar indiretamente na sua introdução (Walker, 2006, 2007). Estas definições também são convergentes com as definições de Tidd, Bessant e Pavitt (1997) e Sundbo (2003), discutidas anteriormente no âmbito de inovações de processo para o setor privado.

Na literatura revisada neste artigo foram identificados três tipos de inovação de processo no setor público: tecnológicas, organizacionais ou administrativas e orientadas ao mercado. Inovações tecnológicas podem ser definidas como novos elementos no sistema de produção de uma organização, incluindo novas tecnologias ou novos sistemas de gestão da informação 
(Walker, 2006, 2007; Damanpour, Walker e Avellaneda, 2009). Inovações organizacionais ou administrativas são novas abordagens e práticas que modificam as relações entre membros da organização e afetam regras, papéis, procedimentos, estruturas, comunicações e relações de troca com o ambiente externo (Walker, 2006, 2007; Damanpour, Walker e Avellaneda, 2009). A inovação de processo orientada ao mercado envolve a modificação de procedimentos e sistemas operacionais da organização para aumentar a eficiência ou a efetividade da produção e a oferta de serviços aos usuários (Walker, 2007).

Aqui se destaca que, em comparação com a literatura sobre inovação no setor privado, há alguma sobreposição dos conceitos. Inovações administrativas ou organizacionais foram destacadas como um tipo independente de inovações de processos por Birkinshaw, Hamel e Mol (2008) e Carayannis, Gonzalez e Wetter (2003). Já Walker (2006, 2007) e Damanpour, Walker e Avellaneda (2009), que estudam a inovação no setor público, entendem esse tipo de inovação como um subconjunto de inovação de processo.

Com o intuito de gerar dados comparáveis com o setor privado, Bloch (2010) apoiou-se na definição do Manual de Oslo (OCDE, 2005) para propor quatro tipos de inovação adequados ao contexto do setor público, sumariados no quadro 3.

Quadro 3

Tipos de inovação no setor público

\begin{tabular}{|ll|}
\hline Tipos de inovação & Definição \\
\hline Inovação de produto & $\begin{array}{l}\text { Introdução de serviço ou bem novo ou significativamente melhorado comparado com os serviços e } \\
\text { bens já existentes na organização. }\end{array}$ \\
Inovação de processo & $\begin{array}{l}\text { Implementação de método de produção ou entrega de serviços ou bens novo ou significativamente } \\
\text { melhorado comparado com os processos já existentes na organização. }\end{array}$ \\
Inovação & Implementação de novo método organizacional ou gerencial que difere significativamente dos métodos \\
organizacional & já existentes na organização. \\
Inovação em & Implementação de novo método de promoção da organização ou de seus serviços e bens, ou novos \\
comunicação & métodos para influenciar o comportamento de indivíduos ou outras organizaçães. \\
\hline
\end{tabular}

Fonte: Adaptado de Bloch (2010:20)

Bloch (2010) argumenta que, embora o uso do Manual de Oslo como referência para o desenvolvimento de indicadores para o setor público se justifique, devido aos anos de experiência na medição da inovação no setor privado, e que há um interesse grande entre stakeholders no sentido de alcançar o máximo de comparabilidade possível com os dados de inovação no setor privado, há ressalvas no que diz respeito à relevância e aos possíveis resultados dessas comparações. O autor afirma que, embora elementos genéricos possam ser similares aos dos ambientes de negócios, existem diferenças importantes em relação a características dos serviços oferecidos, ao processo de tomada de decisão, à estrutura organizacional, às condições gerais, aos objetivos e ao processo como um todo em que as ideias são criadas, transformadas e implementadas como inovações. Isso sugere que, embora o modelo geral possa ser 
similar, quando detalhado, o modelo de medição precisará ser necessariamente diferente para capturar as especificidades da inovação no setor público.

A tipologia proposta por Bloch (2011) foi também utilizada na pesquisa realizada pelo Gallup Organization (2010) com organizações públicas europeias, com a ressalva de que a inovação de produto de Bloch, assim como proposto por Walker (2007), foi ajustada para referir-se apenas à inovação de serviço. Já a pesquisa realizada por Hughes, Moore e Kataria (2011) em organizações públicas do Reino Unido focou inovações de serviços e de processos empresariais. Inovações de serviços incluem expansão da oferta de serviços e melhorias na velocidade de entrega, qualidade, acesso e avaliação dos usuários. Inovações de processos empresariais incluem mudanças na estratégia corporativa e técnicas avançadas de gestão, como sistemas de gestão do conhecimento, investimento em pessoal e mudanças na estrutura organizacional.

Analisando-se os tipos de inovação no setor público estudados recentemente, pode se perceber algumas diferenças e sobreposições entre eles. Walker (2006) e Bloch (2010) foram os únicos que adotaram o conceito de inovação de produto para referir-se a novos bens e serviços. Já a maioria dos autores preferiu focar apenas inovações de serviços, excluindo novos bens de sua definição (The Gallup Organization, 2010; Hughes, Moore e Kataria, 2011; Walker, 2007; Damanpour, Walker e Avellaneda, 2009). Isso pode ser relacionado com o que argumenta Vargas (2010:60), de que inovações, no contexto da gestão pública, "resultam majoritariamente em novos serviços".

Ao comparar as definições de inovações organizacionais (Bloch, 2010; The Gallup Organization, 2010) e de inovações de processo organizacional (Walker, 2006, 2007; Damanpour, Walker e Avellaneda, 2009) pode-se concluir que ambas focam mudanças de estrutura, estratégia e práticas gerenciais. Já as definições de Bloch (2010), Gallup Organization (2010) e Hughes, Moore, Kataria (2011) para inovação de processo parecem englobar as definições de Walker (2006) e Damanpour, Walker e Avellaneda (2009) de inovação de processo tecnológico, incluindo também outras funções de suporte não necessariamente tecnológicas como contabilidade e compras.

Por fim, o quadro 4 apresenta uma síntese dos estudos revisados neste artigo em relação à terceira categoria de análise, descrevendo os principais métodos de pesquisa utilizados e temas examinados.

Quadro 4

Características dos estudos analisados por autor, tema e método

\begin{tabular}{|cclll|}
\hline ID & Ano & \multicolumn{1}{c}{ Autores } & \multicolumn{1}{c|}{ Tema } & \multicolumn{1}{c|}{ Método de pesquisa } \\
\hline 1 & 2011 & Hughes, Moore e Kataria & Medição da inovação & Quantitativa/Survey \\
2 & 2010 & The Gallup Organization & Estratégias de inovação & Quantitativa/Survey \\
3 & 2010 & National Audit Office & Ambiente e estratégias de inovação & Quantitativa/Survey \\
4 & 2010 & Bloch & Medição da inovação & Quantitativa/Survey \\
\hline
\end{tabular}

Continua 


\begin{tabular}{|c|c|c|c|c|}
\hline ID & Ano & Autores & Tema & Método de pesquisa \\
\hline 5 & 2010 & Luke, Verreynne e Kearins & $\begin{array}{l}\text { Indutores e facilitadores de inovações } \\
\text { no setor público }\end{array}$ & Qualitativa/Estudo de Caso \\
\hline 6 & 2010 & Brown & $\begin{array}{l}\text { Gestão de riscos e inovação no setor } \\
\text { público }\end{array}$ & Qualitativa/Estudo de Caso \\
\hline 7 & 2010 & Queiroz e Ckagnazaroff & Inovação em gestão & Qualitativa/Entrevista \\
\hline 8 & 2010 & Ferrarezi, Amorim, Tomacheski & Sustentabilidade de inovações & Quantitativa/Survey \\
\hline 9 & 2010 & Vargas & Disseminação de inovações & Qualitativa/Estudo de caso \\
\hline 10 & 2010 & Hansen & $\begin{array}{l}\text { Antecedentes de inovações } \\
\text { organizacionais }\end{array}$ & Quantitativa/Survey \\
\hline 11 & 2010 & Fernández e Wise & $\begin{array}{l}\text { Preditores da adoção da inovação em } \\
\text { escolas públicas }\end{array}$ & Quantitativa/Survey \\
\hline 12 & 2010 & Walker, Damanpour e Devece & $\begin{array}{l}\text { Adoção de inovação em gestão e } \\
\text { desempenho organizacional }\end{array}$ & Quantitativa/Survey \\
\hline 13 & 2010 & Potts & Inovação no setor público & Ensaio teórico \\
\hline 14 & 2010 & Potts e Kastelle & Inovação no setor público & Ensaio teórico \\
\hline 15 & 2010 & Bommert & Inovação colaborativa & Qualitativa/Estudo de caso \\
\hline 16 & 2010 & Gambarotto e Camozzo & Barreiras à inovação & Quantitativa/Survey \\
\hline 17 & 2010 & Husig e Mann & Liderança e inovação & Qualitativa/Estudo de caso \\
\hline 18 & 2009 & Damanpour e Schneider & $\begin{array}{l}\text { Papel dos gerentes e características da } \\
\text { inovação }\end{array}$ & Quantitativa/Survey \\
\hline 19 & 2009 & $\begin{array}{l}\text { Damanpour, Walker e } \\
\text { Avellaneda }\end{array}$ & $\begin{array}{l}\text { Adoção de inovações e desempenho } \\
\text { em organizações de serviço público }\end{array}$ & Quantitativa/Survey \\
\hline 20 & 2009 & Potts & Inovação no setor público & Ensaio teórico \\
\hline 21 & 2008 & Farah & Disseminação de inovações & Ensaio teórico \\
\hline 22 & 2008 & Vigoda-Gadot et al. & $\begin{array}{l}\text { Inovação no setor público do ponto de } \\
\text { vista do cidadão }\end{array}$ & $\begin{array}{l}\text { Quantitativa e qualitativa/Survey } \\
\text { e entrevistas }\end{array}$ \\
\hline 23 & 2008 & Tolbert, Mossberger e Mcneal & Sustentabilidade de inovações & Quantitativa/Survey \\
\hline 24 & 2007 & Walker et al. & $\begin{array}{l}\text { Tipos de inovação e características } \\
\text { organizacionais e ambientais }\end{array}$ & Quantitativa/Survey \\
\hline 25 & 2007 & Ferrarezi e Amorim & $\begin{array}{l}\text { Características de iniciativas inovadoras } \\
\text { premiadas }\end{array}$ & Qualitativa/Pesquisa documental \\
\hline 26 & 2006 & Damanpour e Schneider & Adoção de inovações & Quantitativa/Survey \\
\hline 27 & 2006 & Walker & Adoção e difusão de inovações & Quantitativa/Survey \\
\hline 28 & 2006 & Asazu & Liderança e inovação & Qualitativa/Pesquisa documental \\
\hline 29 & 2005 & Schwella & Liderança e inovação & Ensaio teórico \\
\hline 30 & 2003 & Lonti e Verma & Determinantes de inovações & Quantitativa/Survey \\
\hline 31 & 2002 & Walker, Jeanes e Rowland & Medição da inovação & Qualitativa/Pesquisa documental \\
\hline 32 & 2001 & Borins & $\begin{array}{l}\text { Características de iniciativas inovadoras } \\
\text { premiadas }\end{array}$ & Quantitativa/Survey \\
\hline 33 & 2001 & Dobbins et al. & Difusão de inovações & Quantitativa/Survey \\
\hline 34 & 2000 & Borins & Liderança e inovação & Quantitativa/Survey \\
\hline
\end{tabular}

Fonte: Elaborado pelas autoras. 
Conforme apresentado no quadro 4, pode-se observar que, entre as pesquisas empíricas, 19 adotaram abordagens quantitativas, nove qualitativas e uma adotou a abordagem mista. Em termos de método de pesquisa, 19 artigos realizaram apenas levantamento de dados (survey), cinco fizeram estudos de caso (um utilizou entrevistas, pesquisa documental e observação, dois, apenas pesquisa documental, e dois, pesquisa documental e entrevista), três fizeram pesquisa documental, um utilizou apenas entrevistas e um realizou levantamento de dados (survey) combinado a entrevistas semiestruturadas.

A análise dos temas estudados nos últimos 10 anos revelou a preferência de pesquisadores por quatro temas: antecedentes da inovação, características gerais, modelos de medição e aspectos relacionados à liderança. Em seguida vêm os temas de disseminação, sustentabilidade e efeitos da inovação no desempenho das organizações públicas.

\section{Conclusões}

A revisão bibliográfica dos estudos sobre inovação no setor público no período de 2000 a 2010 permitiu identificar os principais autores, temas, métodos e resultados encontrados até o momento, facilitando o processo de planejamento de pesquisas futuras.

A análise do referencial teórico das pesquisas revelou que os conceitos de inovação adotados pelos autores guardam semelhança com os já consolidados na literatura sobre inovação no setor privado. A inovação deve incluir produtos, processos e métodos que as organizações desenvolvem de maneira pioneira e aqueles que foram adotados de outras empresas ou organizações. A análise dos estudos revelou, porém, que o setor privado tende a valorizar mais inovações inéditas para o estado da arte, enquanto no setor público as inovações adotadas (geradas originalmente em outro lugar) são mais comuns.

Foi possível observar também que os principais tipos de inovação estudados no setor público são inovação em serviços e processos, e nesse último, a depender do autor, podem estar incluídas inovações em estruturas e sistemas administrativos e inovações de cunho tecnológico.

Entre as pesquisas analisadas, destaca-se o grande número de estudos que buscam identificar antecedentes ambientais, organizacionais e gerenciais que influenciam a inovação no setor público. Outros temas com estudos recentes são características gerais e modelos de medição da inovação no setor público, aspectos relacionados à liderança, disseminação e sustentabilidade de inovações.

No que diz respeito aos aspectos metodológicos, foram encontrados mais estudos empíricos que ensaios teóricos. Entre as pesquisas empíricas, há uma predominância de abordagens quantitativas, com o uso de levantamento de dados (survey). Já nas pesquisas qualitativas, há

equilíbrio no uso de estudos de caso, entrevistas e pesquisas documentais. Apenas um estudo utilizou abordagem mista (levantamento de dados e entrevistas semiestruturadas). 
A análise dos estudos permitiu identificar também lacunas de pesquisa ou temas que necessitam de mais estudos empíricos a fim de promover uma melhor compreensão dos diversos aspectos que compõem um fenômeno complexo como a inovação no setor público. Cinco desses temas são apontados no quadro 5.

\section{Quadro 5}

\section{Proposta de agenda de pesquisa}

\begin{tabular}{|c|c|}
\hline Tema & Sugestão de pesquisa \\
\hline $\begin{array}{l}\text { Processo de } \\
\text { inovação }\end{array}$ & $\begin{array}{l}\text { Estudos comparativos para examinar os efeitos de características ambientais, organizacionais e de } \\
\text { dirigentes nas diferentes fases do processo de inovação em organizações que geram ou adotam } \\
\text { inovações. } \\
\text { v Papel de processos-chave como formulação e conteúdo da estratégia, liderança, comunicação e } \\
\text { resolução de conflitos como mediadores da inovação e do desempenho organizacional. } \\
\text { - Relação entre sustentabilidade de inovações e características da organização. }\end{array}$ \\
\hline $\begin{array}{l}\text { Determinantes, } \\
\text { indutores, } \\
\text { facilitadores e } \\
\text { barreiras }\end{array}$ & $\begin{array}{l}\text { Efeitos do contexto geral político, opinião pública e associação a redes profissionais na geração ou } \\
\text { adoção de inovações. } \\
\text { Fatores que atuam como indutores ou como barreiras nesse processo, estimulando ou impedindo o } \\
\text { desenvolvimento e a implementação de práticas inovadoras. }\end{array}$ \\
\hline Liderança & $\begin{array}{l}\text { Análise multinível combinando a visão de dirigentes e de funcionários de outros níveis da organização } \\
\text { para compreender a inovação no setor público. } \\
\text { Desenvolver e validar escala para auxiliar a determinar que comportamentos de líderes tenham o } \\
\text { maior impacto na probabilidade de inovar. }\end{array}$ \\
\hline Efeitos & $\begin{array}{l}\text { Pesquisas longitudinais para comparar e contrastar as consequências de inovações no desempenho } \\
\text { de organizações públicas e examinar em maior detalhe os períodos de tempo entre a implementação } \\
\text { de inovações e seu impacto no desempenho organizacional. } \\
\text { v Correlacionar a inovação no setor público em diferentes países com indicadores de desempenho para } \\
\text { demonstrar a importância global da inovação e sua singularidade em diversos países. }\end{array}$ \\
\hline Disseminação & - Disseminação de inovações a partir da perspectiva da organização adotante. \\
\hline
\end{tabular}

Fonte: Elaborado pelas autoras.

Entre as limitações do presente estudo, observa-se que os critérios de seleção dos materiais deixaram de fora trabalhos apresentados em congressos, livros e artigos científicos não classificados pela base de dados Qualis 2008 e ISI 2011. Sugere-se, portanto, que futuros levantamentos bibliográficos incluam, por exemplo, livros, dissertações, teses e artigos científicos em outras áreas além da administração a fim de apresentar um panorama mais completo da produção do conhecimento sobre inovação no setor público. 


\section{Referências}

ALBERTI, Adriana; BERTUCCI, Guido. Replicating innovations in governance: an overview. In: ALBERTI, Adriana; BERTUCCI, Guido (Org.). Innovations in governance and public administration: replicating what works. Nova York: United Nations Publication, 2006. cap. 1.

ASAZU, Claudia. Uma conversa sobre liderança. Revista do Serviço Público, Brasília, v. 57, n. 2, p. 259-266, 2006.

BIRKINSHAW, Julian; HAMEL, Gary; MOL, Michael J. Management innovation. Academy of Management Review, v. 33, n. 4, p. 825-845, 2008.

BLOCH, Carter. Measuring public innovation in the Nordic countries: Final Report, 2011. Disponível em: <www.mepin.eu/index.php?option $=$ com_content\&view =article\&id=33:new-pilotstudy \&catid=1:news $>$. Acesso em: 29 jun. 2011.

BLOCH, Carter. Towards a conceptual framework for measuring public sector innovation, 2010. Disponível em: <www.mepin.eu/index.php?option=com_content\&view=article\&id=33:newpilotstudy\&catid=1:news>. Acesso em: 29 jun. 2011.

BOMMERT, Ben. Collaborative innovation in the public service. International Public Management Review, v. 11, n. 1, p. 15-33, 2010.

BORINS, Sandford. Encouraging innovation in the public sector. Emerald: Journal of Intellectual Capital, v. 2, n. 3, p. 310-319, 2001a.

BORINS, Sandford. Leadership and innovation in the public sector. Emerald: Leadership \& Organization Development Journal, v. 23, n. 8, p. 467-476, 2002.

BORINS, Sandford. Loose cannons and rule breakers, or enterprising leaders? Some evidence about innovative public managers. Public Administration Review, v. 60, n. 6, p. 498-507, 2000.

BORINS, Sandford. Public management innovation: toward a global perspective. The American Review of Public Administration, v. 31, n. 5, p. 5-21, $2001 \mathrm{~b}$.

BROWN, Louise. Balancing risk and innovation to improve social work practice. British Journal of Social Work, v. 40, n. 4, p. 1211-1228, 2010.

CARAYANNIS, Elias G.; GONZALEZ, Edgar; WETTER, John J. The nature and dynamics of discontinuous and disruptive innovations from a learning and knowledge management perspective. In: SHAVININA, Larisa V. (Org.). The international handbook on innovation. Oxford: Elsevier Science, 2003. p. 115-138.

DAMANPOUR, Fariborz; SCHNEIDER, Marguerite. Characteristics of innovation and innovation adoption in public organizations: assessing the role of managers. Journal of Public Administration Research and Theory, v. 19, n. 3, p. 495-522, 2009.

DAMANPOUR, Fariborz; SCHNEIDER, Marguerite. Phases of the adoption of innovation in organizations: effects of environment, organization and top managers. British Journal of Management, v. 17 , p. $215-236,2006$. 
DAMANPOUR, Fariborz; WALKER, Richard M.; AVELLANEDA, Claudia N. Combinative effects of innovation types and organizational performance: a longitudinal study of service organizations. Journal of Management Studies, v. 46, n. 4, p. 650-675, 2009.

DOBBINS, Maureen et al. Factors of the innovation, organization, environment and individual that predict the influence five systematic reviews had on public health decisions. International Journal of Technology Assessment in Health Care, v. 17, n. 4, p. 467-478, 2001.

FARAH, Marta F. S. Disseminação de inovações e políticas públicas e espaço local. Revista Organizações \& Sociedade, v. 15, n. 45, p. 107-126, 2008.

FERNÁNDEZ, Sergio; WISE, Lois R. An exploration of why public organizations "ingest" innovations. Public Administration Journal, v. 88, n. 4, p. 979-998, 2010.

FERRAREZI, Elisabete; AMORIM, Sonia. Concurso inovação na gestão pública federal: análise de uma trajetória (1996-2006). Cadernos Enap, Brasília, n. 32, p. 1-53, 2007.

FERRAREZI, Elisabete; AMORIM, Sonia; TOMACHESKI, João. A sustentabilidade de iniciativas premiadas no Concurso Inovação: indícios de mudança da gestão no governo federal? Cadernos Enap, Brasília, n. 34, p. 11-51, 2010.

GALLOUJ, Faiz. Economia da inovação: um balanço dos debates recentes. In: BERNARDES, Roberto; ANDREASSI, Tales (Org). Inovação em serviços intensivos em conhecimento. São Paulo: Saraiva, 2007. cap 1 .

GAMBAROTTO, Francesca; CAMOZZO, Alberto. Dreams of silence: employee voice and innovation in a public sector community of practice. Innovation: Management, Policy \& Practice Journal, v. 12, n. 2, p. 166-179, 2010.

HANSEN, Morten B. Antecedents of organizational innovation: the diffusion of new public management into Danish local government. Public Administration Journal, 2010. Disponível em: < http:// onlinelibrary.wiley.com/doi/10.1111/j.1467-9299.2010.01855.x/abstract>. Acesso: em 22 out. 2010.

HUGHES, Alastair; MOORE, Kyla; KATARIA, Nimesh. Innovation in public sector organisations: a pilot survey for measuring innovation across the public sector. Londres, 2011. Disponível em: $<$ www.nesta.org.uk/aboutus/assets/features/innovation_in_public_sector_organisations $>$. Acesso em: 28 jun. 2011.

HUSIG, Stefan; MANN, Hans-George. The role of promoters in effecting innovation in higher education institutions. Innovation: Management, Policy \& Practice, v. 12, n. 2, p.180-191, 2010.

KEARNEY, Richard C.; FELDMAN, Berry M.; SCAVO, Carmine F. Reinventing government: city manager attitudes and actions. Public Administration Review, v. 60, n. 6, p. 535-547, 2000.

LONTI, Zsuzsanna; VERMA, Anil. The determinants of flexibility and innovation in the government workplace: recent evidence from Canada. Journal of Public Administration Research and Theory, v. 13, n. 3, p. 283-310, 2003. 
LUKE, Belinda; VERREYNNE, Martie-Louise; KEARINS, Kate. Innovative and entrepreneurial activity in the public sector: the changing face of public sector institutions. Innovation: Management, Policy \& Practice, v. 12, n. 2, 2010. p. 138-153.

MULGAN, Geoff; ALBURY, David. Innovation in the public sector. Londres, v. 1.9, 2003. Disponível em: <http://michaellittle.org/documents/Mulgan\%20on\%20Innovation.pdf>. Acesso em: 25 maio 2010.

NATIONAL AUDIT OFFICE. Achieving innovation in central government organization. Londres, 2006. Disponível em: <www.official-documents.gov.uk/document/hc0506/hc14/1447/1447.pdf> . Acesso em: 3 set. 2011.

OCDE. Manual de Oslo. Diretrizes para coleta e interpretação de dados sobre inovação. 3. ed. Brasília: Finep, 2005.

POTTS, Jason. Innovation by elimination: a proposal for negative policy experiments in the public sector. Innovation: Management, Policy \& Practice Journal, v. 12, n. 2, p. 238-248, 2010.

POTTS, Jason. The innovation deficit in public services: The curious problem of too much efficiency and not enough waste and failure. Innovation: Management, Policy \& Practice Journal, v. 11, p. 3443, 2009.

POTTS, Jason; KASTELLE, Tim. Public sector innovation research: what's next? Innovation: Management, Policy \& Practice, v. 12, n. 2, p. 122-137, 2010.

QUEIROZ, Roberta G. M.; CKAGNAZAROFF, Ivan B. Inovação no setor público: uma análise do choque de gestão (2003-2010) sob a ótica dos servidores e dos preceitos teóricos relacionados à inovação no setor público. Revista de Administração Pública, v. 44, n. 3, p. 679-705, 2010.

ROGERS, Everett M. Diffusion of innovation. Nova York: The Free Press, 1995.

SCHUMPETER, Joseph A. Teoria do desenvolvimento econômico: uma investigação sobre lucros, capital, crédito, juro e o ciclo econômico. São Paulo: Abril Cultural, 1982.

SCHWELLA, Erwin. Inovação no governo e no setor público: desafios e implicações para a liderança. Revista do Setor Público, v. 56, n. 3, p. 259-276, 2005.

SUNDBO, Jon. Innovation and strategic reflexivity: an evolutionary approach applied to services. In: SHAVININA, Larisa V. (Org.). The International Handbook on Innovation. Oxford: Elsevier Science, 2003. p. 97-114.

THE GALLUP ORGANIZATION. Innobarometer, 2010. Analytical report: innovation in public administration, 2011. Disponível em: <http://cordis.europa.eu/ innovation/en/policy/innobarometer. htm. Acesso em: 4 set. 2011.

TIDD, Joe; BESSANT, John; PAVITT, Keith. Managing innovation: integrating technological, market and organizational change. West Sussex: John Wiley \& Sons, 1997.

TOLBERT, Caroline; MOSSBERGER, Karen; MCNEAL, Ramona. Institutions, policy innovation and e-government in the American States. Public Administration Review, v. 68, n. 3, p. 549-563, 2008. 
VAN DE VEN, Andrew H.; ANGLE, Harold L.; POOLE, Marshall S. An introduction to the Minnesota innovation research program. In: VAN DE VEN, Andrew Harold; ANGLE, H. L.; POOLE, Marshall S. (Org.). Research on the management of innovation. The Minnesota studies. Nova York: Oxford University Press, 2000. cap. 1.

VARGAS, Eduardo R. Disseminação de iniciativas inovadoras premiadas no Concurso Inovação na Gestão Pública Federal (1996-2006). Cadernos Enap, Brasília, n. 34, p. 58-115, 2010.

VIGODA-GADOT, Eran et al. Public sector innovation for Europe: a multinational eight-country exploration of citizen's perspectives. Public Administration Journal, v. 86, n. 2, p. 307-329, 2008.

WALKER, Richard M. An empirical evaluation of innovation type and diffusion: an empirical analysis of local government. Public Administration, v. 84, n. 2, p. 311-335, 2006.

WALKER, Richard M. An empirical evaluation of innovation types and organizational and environmental characteristics: towards a configuration framework. Journal of Public Administration Research and Theory, v. 18, n. 4, p. 591-615, 2007.

WALKER, Richard M.; DAMANPOUR, Fariborz; DEVECE, Carlos A. Management innovation and organizational performance: the mediating effect of performance management. Journal of Public Administration Research and Theory, 2010. Disponível em: < http://jpart.oxfordjournals.org/content/early/2010/08/18/jopart.muq043.full.pdf+html>. Acesso em: 4 dez. 2010.

WALKER, Richard; JEANES, Emma; ROWLAND, Robert. Measuring innovation: applying the literature-based innovation output indicator to public services. Public Administration Journal, v. 80, n. 1, p. 201-214, 2002.

Soraya Monteiro Brandão é especialista em políticas públicas e gestão governamental (EPPGG) na Escola Nacional de Administração Pública (Enap). E-mail: sorayamonteiro@hotmail.com.

Maria de Fátima Bruno-Faria é professora do Programa de Pós-Graduação em Administração da Universidade de Brasília (PPGA/UnB). E-mail: fatimabruno@unb.br. 